\title{
Research on Hydrodynamic Performance of Rotatable Cable Underwater Robot
}

\author{
Geng Yu, Zhidong Wang, Hongjie Ling
}

\begin{abstract}
The ROV system dynamics model is established. Hydrodynamic performance of six degrees of freedom of the ROV underwater ontology and umbilical cable under different working conditions are calculated based on CFD numerical simulation method. Preliminary simulation calculation of coupling motion and dynamics between ROV ontology muti propellers during straight navigation and roll motion is carried out based on multi domain flow field data transfer through sliding mesh method, verifing the feasibility of the multi thruster ROV motions and dynamics numerical prediction method.
\end{abstract}

Index Terms - remotely operated vehicle, rov, hydrodynamic performance, interaction

\section{INTRODUCTION}

ROV is an underwater vehicle. The sportiness such as speed and maneuverability is one of the most important parameters of the whole system. At present, the research methods of the hydrodynamic characteristics of ROV mainly include calculation based on theoretical or empirical formulas, maneuverability tests and CFD numerical simulations. The hydrodynamic coefficient of ROV is estimated by a large number of ship model test data analysis and correction formula [1-3]. Maneuverability testing is the only reliable and relatively accurate method for obtaining hydrodynamic coefficients, but high test costs, long test cycles and demanding requirements for test equipment are not conducive to the development of low-cost underwater robots. With the continuous improvement and optimization of numerical solution methods and the rapid changes of computer performance, the numerical calculation of underwater robots based on computational fluid dynamics technology has become a commonly used method for hydrodynamics. Alexander Phillips and Maaten Furlong [4-5] used CFD software to study the resistance performance of three existing underwater robots. The calculation results are consistent with the experimental data, indicating that CFD software can be used underwater. Robot shape concept design and optimization. Quan Wei-cai [6] proposed an accurate geometric finite element model for two-dimensional dynamic analysis of umbilical cable.

Geng Yu Postgraduate of Naval Architecture and Ocean Engineering department of Jiangsu University of Science and Technology, China.+8615262913230

Zhidong Wang, Professor, doctoral tutor, Mainly Engaged in Research Work on Fluid Dynamic Performance of Marine and Offshore Engineering, China.

Hongjie Ling Lecturer Jiangsu University of Science and Technology School of Naval Architecture and Ocean Engineering

\section{ROV STRUCTURE BODY HYDRODYNAMIC PERFORMANCE CALCULATION}

A relatively reliable and accurate method for measuring the performance of underwater robots is to conduct maneuverability tests on underwater robots, but this method requires high time and economic cost and is not suitable for the development of small underwater robots. With the advancement of technology and the continuous improvement of computer performance, the use of CFD software to study the performance of underwater robots has become a common means of hydrodynamic performance calculation in the initial stage of prototype development. This chapter will conduct preliminary numerical calculation and analysis of hydrodynamic performance and fully coupled motion simulation of reversible ROV based on fluid dynamics simulation software FINE/Marine.

\section{A. Model processing and meshing}

The ROV body has a complex structure and many components, and many small parts are difficult to mesh. Therefore, before importing CFD software for calculation, the model needs to be simplified, that is, without changing the overall structure of the ROV body, some elements such as edges, corners, holes, etc., which have little influence on the hydrodynamic performance, are removed, and the ROV simplified model is as follows. Figure 1 shows.

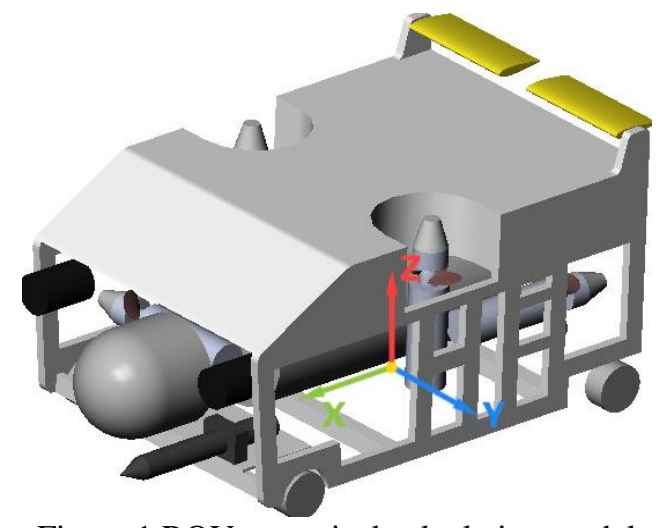

Figure 1 ROV numerical calculation model

The 3D model was completed using SolidWorks software and imported into Hexpress software for meshing. The calculation domain is a $9 \mathrm{~L} \times 4 \mathrm{~L} \times 4 \mathrm{~L}$ cuboid domain, the ROV body head is $3 \mathrm{~L}$ from the entrance, and $\mathrm{L}$ is the ROV body length. The grid type adopted is a full-hexahedral unstructured grid, which follows the principle of overall unstructured and local encryption. After many optimizations, the final grid number is about 1.13 million. The grid division of ROV hydrodynamic calculation model is shown in Figure 2. 


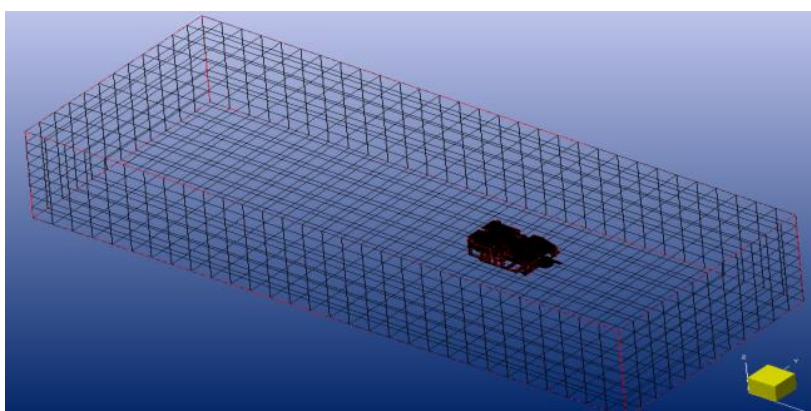

Figure 2 Computational model meshing

\section{B. Forward direct hydrodynamic calculation}

In the numerical simulation software, the fluid parameters were set to $20{ }^{\circ} \mathrm{C}$ fresh water, the density was $998.2 \mathrm{~kg} / \mathrm{m} 3$, and the dynamic viscosity coefficient was $0.001002 \mathrm{pa} \cdot \mathrm{s}$; the $\mathrm{k}-\omega$ turbulence model was used for all working conditions. The resistance $\mathrm{R}$, the vertical force $\mathrm{Fz}$, and the pitching moment $\mathrm{My}$, which are subjected to the ROV sailing at a speed of $0.5 \mathrm{kn}$ to $5 \mathrm{kn}$, are calculated respectively. The specific results are shown in Figs. 3 to 5.

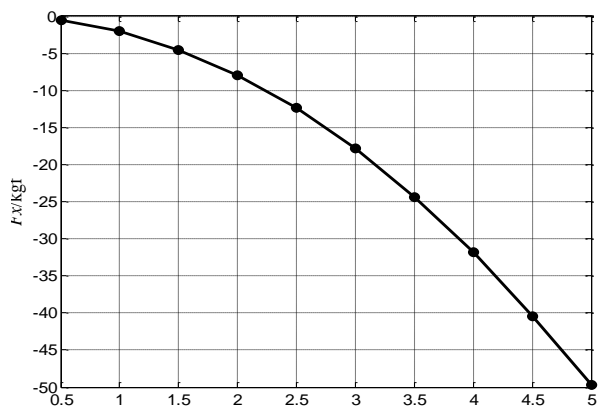

Figs. 3 Resistance to the body

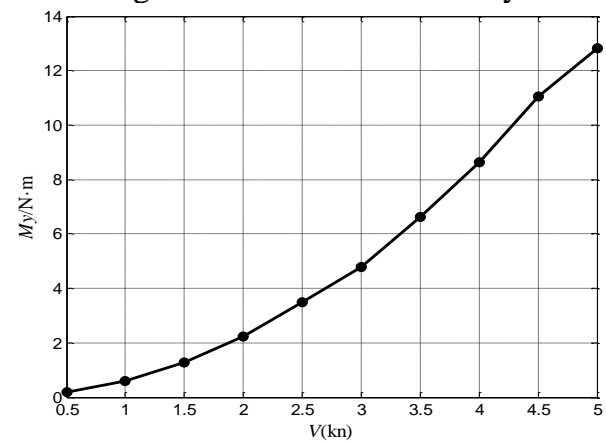

Figs. 4 Vertical force

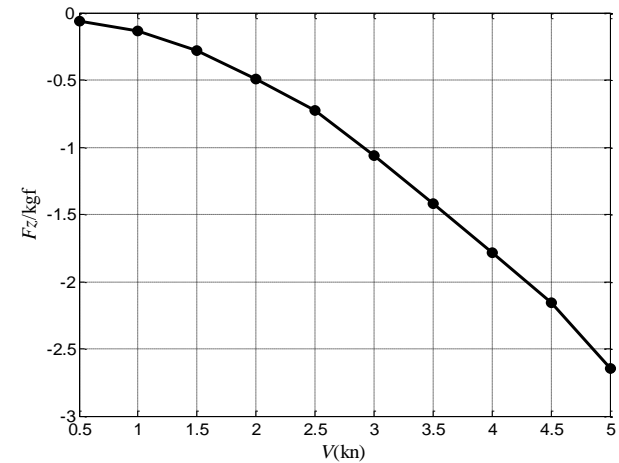

Figs. 5 Pitch moment

At the design speed of $4 \mathrm{kn}$, the longitudinal resistance is approximately $32 \mathrm{kgf}$, and the highest thrust ( $34 \mathrm{kgf}$ ) that the propeller can provide meets the requirements for use. The pressure cloud at this speed is shown in Figure 6.

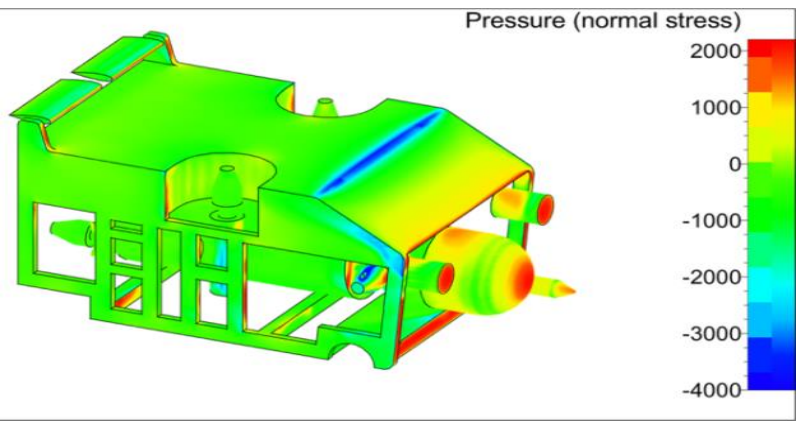

Fig.6 Pressure cloud of straight navigation

It can be seen from the pressure cloud diagram that the slope of the front end of the floating body causes the ROV to be subjected to the downward pressure of the Z-axis in the direct flight process. The $\mathrm{FZ}$ reaches $1.8 \mathrm{kgf}$ at $\mathrm{V}=4 \mathrm{kn}$; the means to solve this problem is to use the depth control. The algorithm controls the vertical thruster to generate a reverse thrust according to the feedback signal of the depth gauge to keep the ROV sailing at a specified depth.

Due to the structural asymmetry, the pressure is concentrated on the upstream surface of the lower part of the ROV, causing it to experience a large downturn moment during direct flight. When $\mathrm{V}=4 \mathrm{kn}$, My reaches $8.5 \mathrm{~N} \cdot \mathrm{m}$, which affects the navigation stability. In order to eliminate the influence of My, a hydrofoil is placed at the tail of the body to provide the $\mathrm{Z}$-axis negative lift, which produces mutually canceling torque. Using the second power function to fit the velocity VX and the lower depression moment My can be approximated as follows:

$$
M_{y}=1.844 V_{X}^{2}+0.3773 V_{X}-0.0576 \quad \text { Taking }
$$

into account the above calculated safety factor, it is decided to set the wing angle of attack to $7^{\circ}$, at which time the lift coefficient $\mathrm{Cz}=0.42$, and the hydrofoil module can provide a harmonic torque of $8.5 \mathrm{~N} \cdot \mathrm{m}$ to ensure the stability of the ROV direct motion. The velocity-harmonic torque curve thus obtained is shown in Fig. 7 and Fig. 8. It can be seen that the blending moment MY is in good agreement with the downturn moment My curve, indicating that the hydrofoil angle of attack is suitable for full speed conditions.

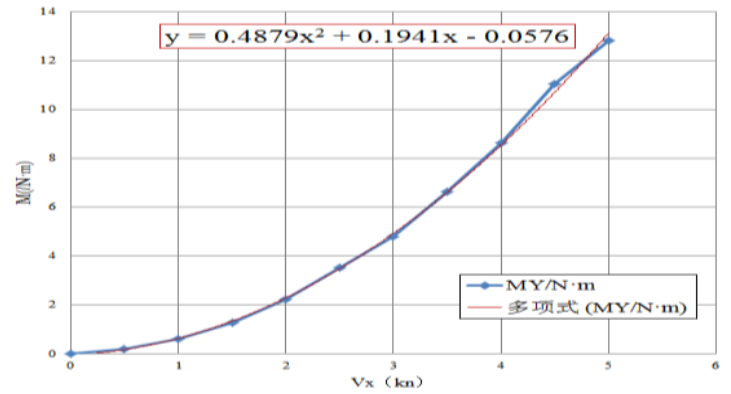

Fig.7 $\mathrm{V}_{\mathrm{X}}-\mathrm{M}_{\mathrm{Y}}$ fitting curve

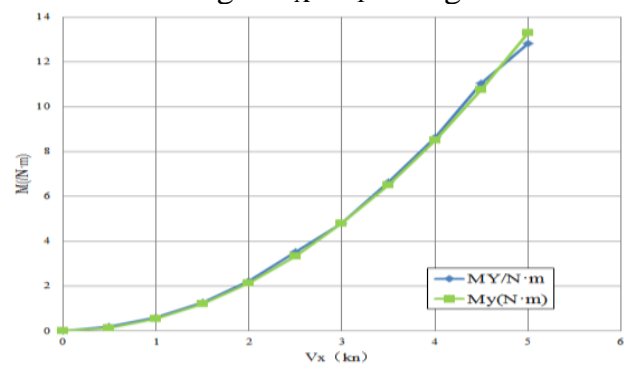

Fig. $8 \mathrm{~V}_{\mathrm{X}}-\mathrm{M}_{\mathrm{Y}}$ fitting curve 
The principle of harmonic adjustment and the best angle of attack are shown in Figure 9 and Figure 10.



Fig.9 Hydrofoil harmonic principle NACA0018 $\lambda=2.5$

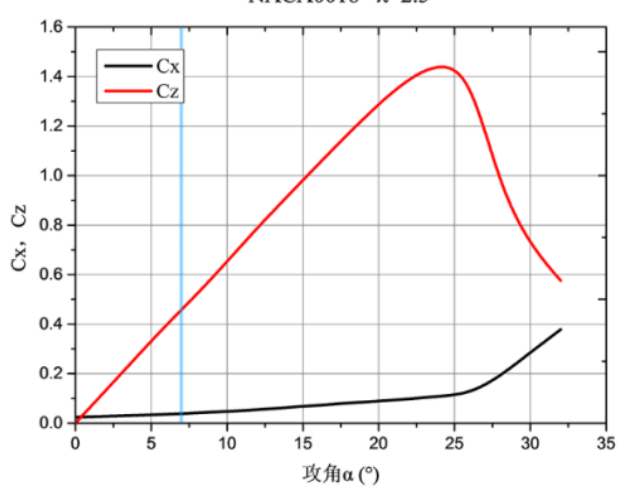

Fig.10Attack angle selection

\section{Flip hydrodynamic calculation}

The flipping action of the ROV around the $\mathrm{X}$ axis is achieved by the rotation of the opposite thrusters of the two vertical thrusters, resulting in a rotational moment. As shown in Figure 11. This function allows the ROV to maintain an arbitrary roll angle and, with the lateral thruster, it can be more easily attached to the surface of the underwater structure. After the wall is attached, the vertical thruster outputs the same thrust, and the ROV body can be adsorbed on the surface of the hull. By relying on the thrust of the longitudinal thruster and the friction of the wheel set, it is possible to drive forward and backward. By using the differential or reverse of the longitudinal thruster and the universal wheel set module, the flexible steering during the driving process can be realized. The attached wall is shown in Figure 12.

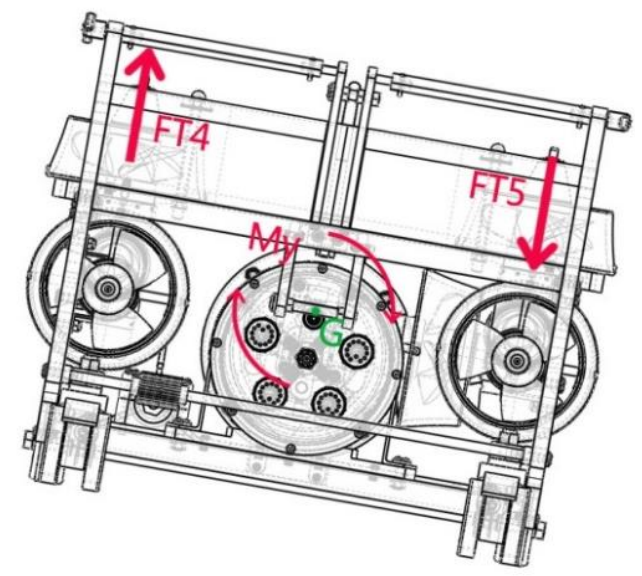

Fig.11 Schematic of rolling around $\mathrm{X}$ axis

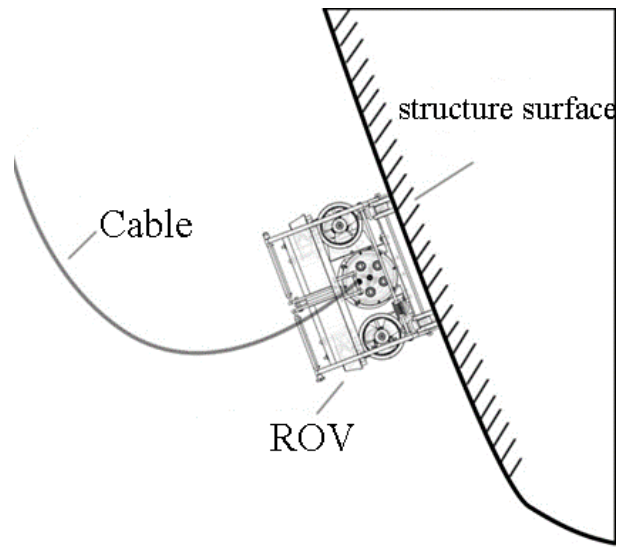

Fig.12 Sticking work diagram

The roll moment Mx, the heading moment My and the heading moment $\mathrm{Mz}$, which the ROV receives when it is turned around the $\mathrm{X}$ axis at an angular velocity of $0.5 \mathrm{rad} / \mathrm{s}$ to $3.0 \mathrm{rad} / \mathrm{s}$. Their results are shown in Figure 13, 14, 15.

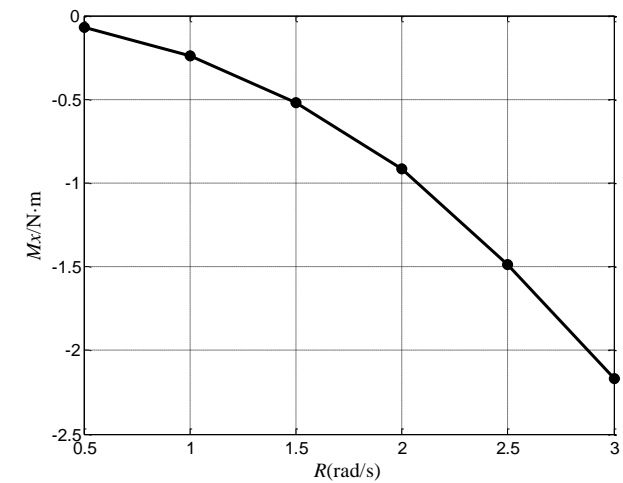

Figure 13 Rolling moment Mx

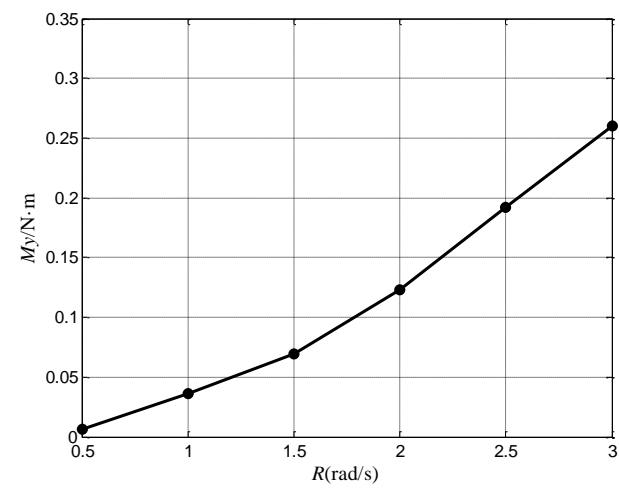

Figure 14 Pitch moment My

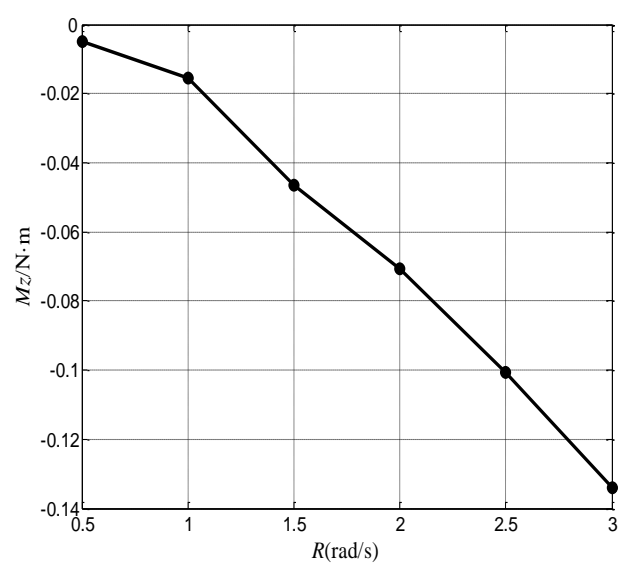

Figure 15 Turning torque $\mathrm{Mz}$ 
When the ROV is turned clockwise around the $\mathrm{X}$ axis at a constant angular velocity of $3 \mathrm{rad} / \mathrm{s}$, a stable resistance torque $\mathrm{Mx}=2.2 \mathrm{~N} \cdot \mathrm{m}$ is obtained. But at the same time, it will be subjected to the positive yaw force of the $\mathrm{Y}$ axis and the negative downward pressure of the $\mathrm{Z}$ axis. This phenomenon is caused by the asymmetry of the ROV structure leading to the unequal pressure distribution of the upper and lower and left and right surfaces when flipping. Relying on its own buoyancy, the vertical propeller is differentially rotated by the depth and navigation control program, and the lateral thruster can be used to ensure its in-place reversal.

Figure 16 shows the comparison of the dynamic torque and the total resistance torque when the ROV is turned over the vertical thruster and then rotated around the $\mathrm{X}$-axis at $1 \mathrm{rad} / \mathrm{s}$ and $3 \mathrm{rad} / \mathrm{s}$. It can be seen from the figure that when the two vertical propellers reach the theoretical maximum thrust of $80 \mathrm{~N}$, the dynamic moment is constant $32.4 \mathrm{~N} \cdot \mathrm{m}$, which is always greater than the total resistance torque, which proves that the ROV can be flipped around the $\mathrm{X}$ axis at a speed of at least $3 \mathrm{rad} / \mathrm{s}$. The time required to complete a flip cycle $(2 \pi \mathrm{rad})$ is $2.09 \mathrm{~s}$. However, in actual operation, in order to avoid the influence of inertia and accurately control the flip angle of the ROV, the angular velocity should not exceed $1 \mathrm{rad} / \mathrm{s}$.

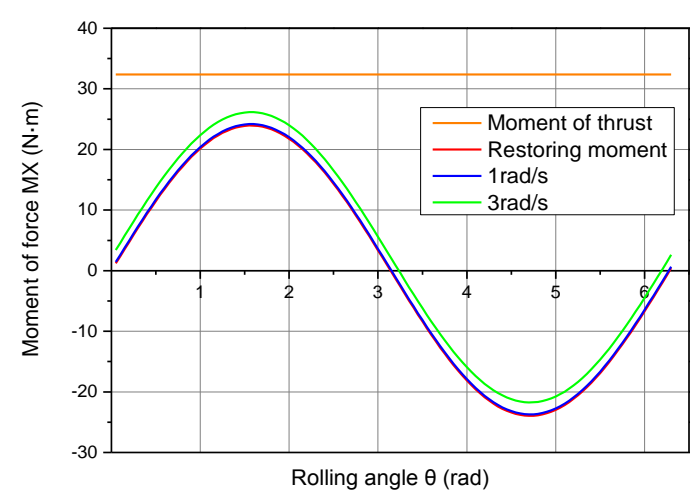

Fig.16 ROV resultant torque about $\mathrm{X}$ axis

\section{ROV BODY-PROPELLER COUPLING MOTION CALCULATION}

The ROV ontology-propeller coupled motion simulation calculation model is a self-propelled model, ie no mandatory motion constraints are imposed on it. The initial model is static, the power input value is the propeller speed, and the blade rotation is driven to generate the thrust; the ROV body generates six-degree-of-freedom motion under the action of the blade thrust and the fluid; since the propeller is hingedly connected with the ROV body, the motion is Affecting the blade thrust, thereby changing the motion state of the entire system, and realizing the motion coupling of the rigid body and the propeller.

\section{A. Sliding grid principle}

Based on the constraint model, the hydrodynamic performance of ROV underwater body is studied in the previous section, which provides a reference for its improved design. In this section, the ROV ontology is divided into computational domains with different motion states through multiple computational domain and slip mesh methods. Slip grid technology is used to transfer flow field information between the contact faces of each computational domain. In the meshing, the intersecting mesh nodes do not need to be completely fitted, and the flow information of each region is transmitted by calculating the flux on both sides of the interface. The calculation principle is shown in Figure 17. The flow field information of unit 1 and unit 2 is transferred to unit 3 through $d-b$ and $b-e$, and the flow field information of unit 3 and unit 4 is transmitted through c-e and b-e. Go to unit 2 . It can be seen that in the calculation, the flux is calculated at the coincidence surface of the two regions rather than at the boundary of the unit itself.

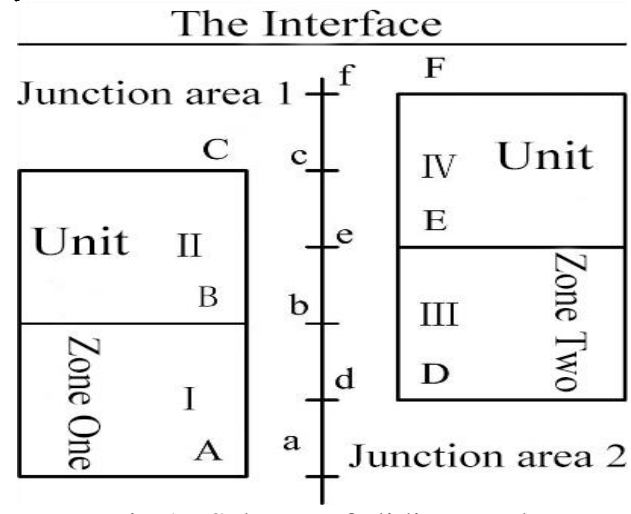

Fig.17 Scheme of sliding mesh

\section{B. Calculation model}

The use of the slip grid method requires strict pre-processing of the computational model. The ROV underwater body consists of an ROV frame body and five propellers of the same type. In order to simulate the actual situation, the model needs to be divided into a rigid body and five rotating moving bodies, five of which include the rotating part of the ROV entity when sailing, ie the blades, hub and fairing of the propeller; In addition to this, the ROV body portion is a rigid body. In order to improve the success rate of the generated grid and reduce the computational time, the frame ontology model is appropriately simplified to remove the small parts and partial pores of the surface. The simplified rigid body model is shown in Fig. 18. The model of the rotational motion part is shown in Fig. 19.



Fig.18 ROV rigid body model

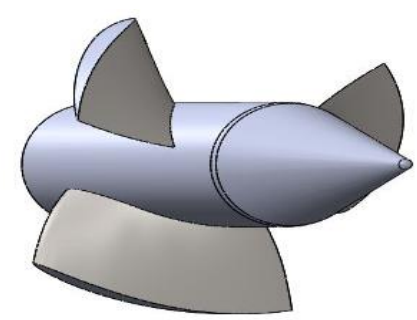

Fig 19 Propeller blade model 
The propeller propeller is a custom small ducted propeller, and the blade parameters are shown in Table 1.

Tab.1 Main parameters of propeller

\begin{tabular}{|l|l|}
\hline PARAMETER & NUMERICAL VALUE \\
\hline Number of blades & 3 \\
\hline Profile shape & NACA66+a $=0.8$ \\
\hline Pitch ratio & 0.85 \\
\hline Disk ratio & 0.90 \\
\hline Roll angle $/^{\circ}$ & 32.0 \\
\hline Paddle diameter / $\mathrm{m}$ & 0.10 \\
\hline Hub diameter ratio & 0.40 \\
\hline
\end{tabular}

C. Computational domain and grid parameter settings

The computational model is divided into six computational domains, including five cylindrical paddle domains and one rectangular outer domain. The boundary of the cylinder paddle is the slip surface. Unstructured meshes are generated for all computational domains by HEXPRESS, and additional encryption is required at the fine faces. The grid distribution of the cylindrical paddle field and the rectangular outer body domain is shown in Figure 20-21.

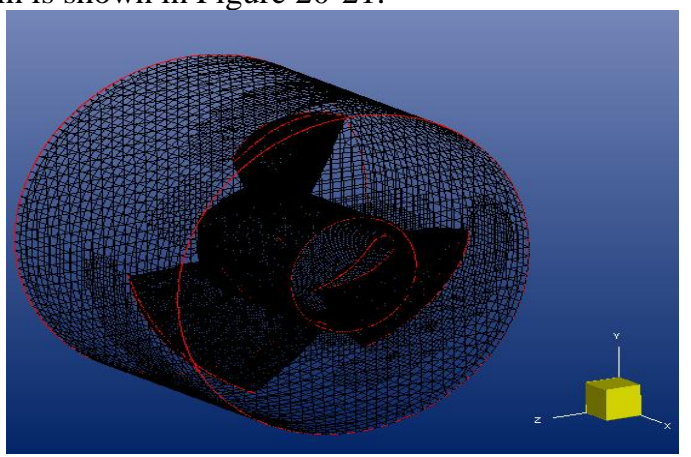

Fig.20 Cylinder domain

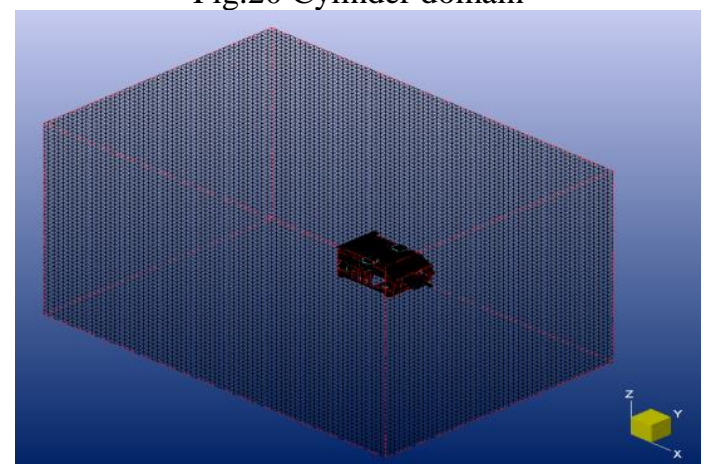

Fig.21 Outside box domain

The final calculation model can be generated by combining the six computational domains defined above. The total number of grid cells is 4442798. As shown in Figure 22.

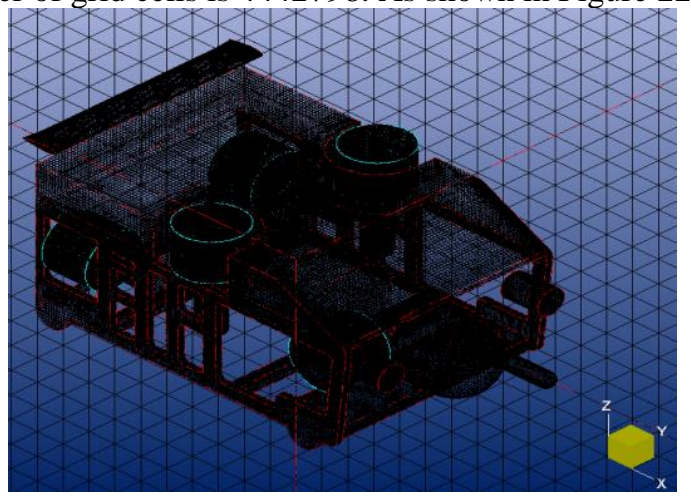

Fig.22 Multi - domain ROV model

\section{ROV direct movement and dynamic calculation}

The movement speed and hydrodynamics of the ROV body in all directions when the two rear propellers rotate at $500-3000 \mathrm{r} / \mathrm{min}$ are calculated. The speed interval is $500 \mathrm{r} / \mathrm{min}$, and the acceleration is accelerated. The time step is $0.004 \mathrm{~s}$ and a total of 5000 steps are calculated.

ROV will perform direct flight movement under this calculation condition. Considering that the physical prototype has automatic heading correction function, in order to get closer to the actual situation and speed up the calculation convergence speed, the traverse, roll and sway degrees of freedom are closed. Straight flight, heave and pitch three-degree-of-freedom motions are solved.

The calculation results show that the propeller blade motion can realize the ROV direct flight motion. As the blade speed increases, the speed and resistance increase. A slight lifting moment will occur during the advancement, resulting in a pitch angle and an upward trend during ROV sailing. The relationship between the speed of direct flight and the relationship between hydrodynamics and blade speed is shown in Figure 23-25

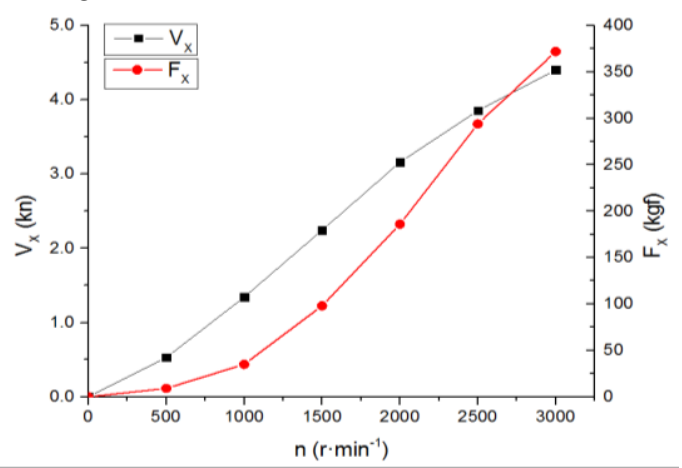

Fig23 Vertical propulsion speed

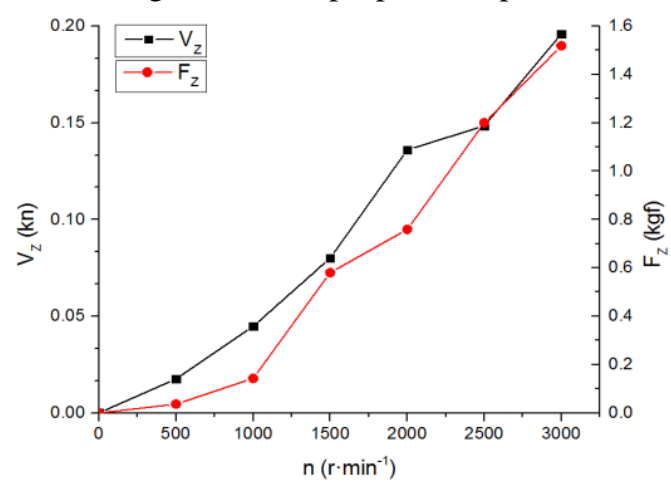

Fig24 Vertical floating speed

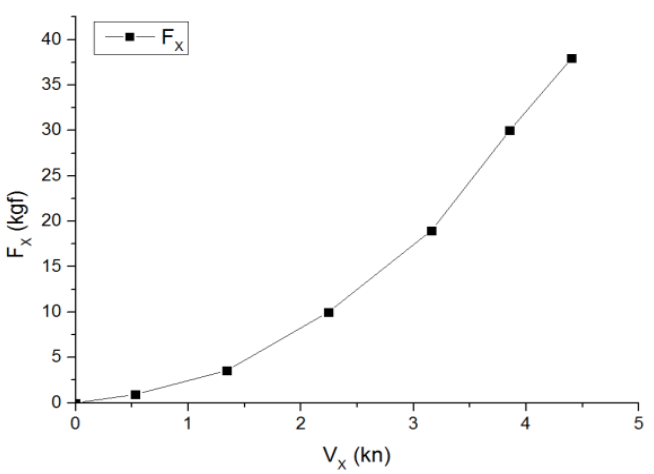

Fig.25 Hydrodynamic - longitudinal velocity

According to the calculation results of the hydrodynamic FX and longitudinal velocity VX fitting curves and the rigid 
model calculations in Section 4.3, it is found that the coupling simulation results show that the resistance is too large and the comprehensive error is about $9.5 \%$. According to the analysis, the coupling calculation model is accompanied by five propeller blades. The leaves create additional resistance. The comparison results are shown in Table 2.

Tab.2 Comparison of direct hydrodynamics between rigid model and coupled model

\begin{tabular}{|l|l|l|l|}
\hline $\begin{array}{l}\text { Direct } \\
\text { speed } \\
\mathrm{V}_{\mathrm{X}}(\mathrm{kn})\end{array}$ & $\begin{array}{l}\text { Rigid model } \\
\text { hydrodynamic } \\
\mathrm{F}_{\mathrm{X} 0}(\mathrm{~N})\end{array}$ & $\begin{array}{l}\text { Coupling model } \\
\text { hydrodynamic } \\
\mathrm{F}_{\mathrm{X}}(\mathrm{N})\end{array}$ & Deviation \\
\hline 1.0 & 2.25 & 2.47 & $9.7 \%$ \\
\hline 2.0 & 8.10 & 8.81 & $8.8 \%$ \\
\hline 3.0 & 17.76 & 19.55 & $10.1 \%$ \\
\hline 3.5 & 24.83 & 27.03 & $8.9 \%$ \\
\hline
\end{tabular}

\section{E. ROV flip motion calculation}

Since the calculation time of each working condition is up to 70 hours, this section calculates the moving speed and hydrodynamics of the ROV body in all directions when the two vertical propellers rotate at $1500 \mathrm{r} / \mathrm{min}$. Use slow acceleration. The time step is $0.004 \mathrm{~s}$ and a total of 5000 steps are calculated. In this calculation condition, the two vertical thrusters will output the thrust in the opposite direction. In order to speed up the calculation of the convergence speed and reduce the error, the pitch, pitch and roll degrees of freedom are closed, and the traverse, heave and cross are Shake three degrees of freedom motion to solve.

The calculation results show that the ROV achieves a continuous inversion motion around the $\mathrm{X}$-axis under this condition. It takes about $4.5 \mathrm{~s}$ from the start of the blade motion to the ROV body to reach the stable inversion speed, and finally stabilizes the flip angular velocity $\omega X=1.79 \mathrm{rad} / \mathrm{s}$. Speed and total The flip angle duration curve is shown in Figure 26. During the steady flipping process, the ROV body hydraulic torque is $\mathrm{My}=4.46 \mathrm{~N} \cdot \mathrm{m}$, and the duration curve is shown in Fig. 27.

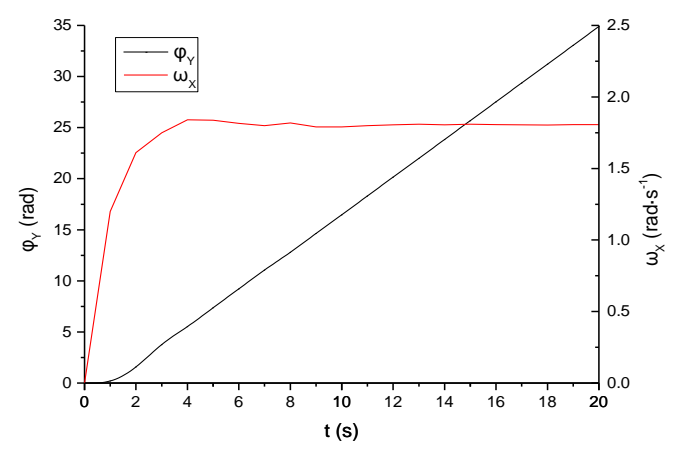

Fig.26 Rolling angular velocity duration

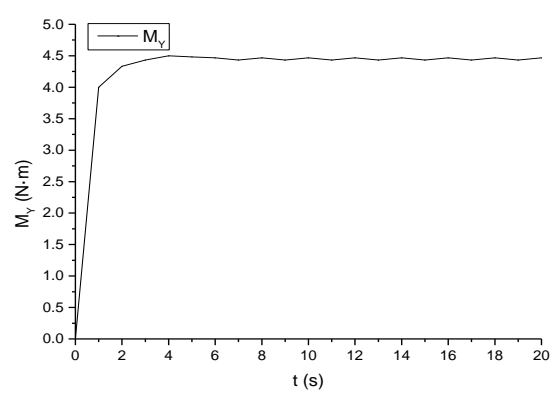

Fig.27 Rolling hydrodynamic moment duration
Since the ROV body structure is not completely symmetrical, and the output thrusts of the two thrusters are inconsistent, the streamline distribution during the ROV inversion process does not exhibit central symmetry, as shown in Figure 28. The ROV's center of gravity motion trajectory is drawn by the ROV center of gravity position. It is found that the flip motion trajectory is not a perfect circle due to the above reasons, and the flip radius is about $1.5 \sim 1.8 \mathrm{~m}$. The trajectory of the ROV center of gravity from the initial position within 10s is shown in Fig. 29.

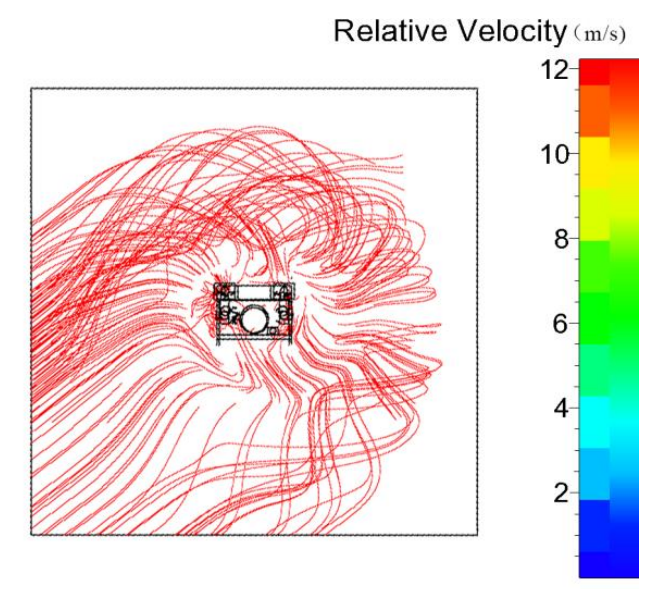

Fig.28 Streamline distribution of rolling ROV

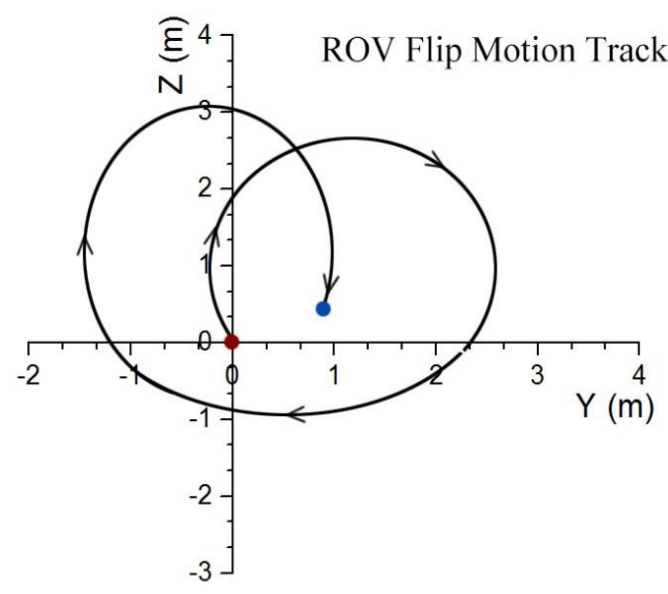

Fig.29 Center of gravity trajectory of ROV

\section{CONCLUSION}

In this thesis, the umbilical cable and the dynamic model of the dynamics of the ROV system are established. The six-degree-of-freedom hydrodynamic performance of the ROV underwater body at different speeds is calculated based on the CFD numerical simulation method. The results show that the ROV dynamic performance is good, and The hydrofoil is adjusted according to the calculation results to further optimize its navigation stability.

The slipping grid and multi-computation domain method are used to simulate the motion and dynamics of the coupling between the ontology and the multi-blade during the direct and reverse motion of the ROV ontology. The results show that this multi-propeller ROV motion is closer to the actual situation. The numerical prediction method has certain feasibility, and provides a reference for the numerical simulation of fully coupled motion of complex structure aircraft such as ROV system. 


\section{REFERENCES}

[1] Deng Wei. Research on 3D motion sliding mode control of underwater robot with cable remote control [D]. Guangzhou: South China University of Technology, 2011.

[2] Wu Jiaming, Yu Miao, Zhu Linlin. Hydrodynamic Mathematical Model and Rotational Motion Analysis of a Remotely Controlled Underwater Vehicle with Cables[J]. Journal of Ship Mechanics, 2011, 15(8): 827-836.

[3] Divine Maalouf, Ahmed Chemorin, Vincent Creuze. Adaptive depth and pitch control of an underwater vehicle with real-time experiments [J]. Ocean Engineering, 2015(98):66-77.

[4] Azis, F.A, Aras, M. S. M, Rashid, Problem Identification for Underwater Remotely Operated Vehicle(ROV) A Case Study[J]. Procedia Engineering, 2012(41):554-560.

[5] Alexander Phillips, Maaten Furlong, Stephen R. Turnock. The Use of Computational Fluid Dynamics to Assess the Hull Resistance of Concept Autonomous Underwater Vehicles [C]. Oceans 2007-Europe, 2007:1292-1297.

[6] QUAN Wei-cai, ZHANG Zhu-ying, ZHANG Ai-qun. Dynamics analysis of planar armored cable motion in deep-sea ROV system[J]. Journal of Central South University, 2014(12):4456-4467.

Geng Yu Postgraduate of Naval Architecture and Ocean Engineering department of Jiangsu University of Science and Technology, China.+8615262913230

Zhidong Wang, Professor, doctoral tutor, Mainly Engaged in Research Work on Fluid Dynamic Performance of Marine and Offshore Engineering, China.

Hongjie Ling Lecturer Jiangsu University of Science and Technology School of Naval Architecture and Ocean Engineering 